Stefan Kubica, Hagen Ringshausen, Jörg Reiff-Stephan, Marius Schlingelhof (Hrsg.) 2. Automobil Symposium Wildau:

Tagungsband Technische Hochschule Wildau 2017

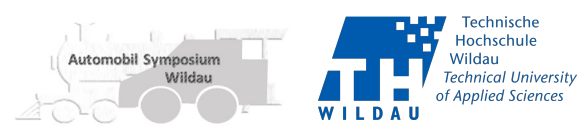

Sebastian Scbulz.

Deutsches Zentrum für Luft- und Raumfahrt e.V.

s.scbul@dlr.de

\title{
Entwicklung eines kostengünstigen smarten Sensors zur Emissionsmessung im urbanen Raum
}

\section{Zusammenfassung}

Die Reduzierung der Emissionsbelastung im urbanen Raum wird immer bedeutender. Einerseits muss der Lebensraum für den Menschen erhalten bleiben, andererseits hat die Luftverschmutzung auch auf andere Regionen der Welt einen starken Einfluss. In Berlin betreibt die Senatsverwaltung vereinzelt Messeinrichtungen zur Untersuchung und Aufzeichnung von Umweltgrößen, wie z.B. der Schadstoffbelastung der Luft. Diese Einrichtungen sind sehr groß und teuer. Um flächendeckende Langzeitmessungen in einer Region zu ermöglichen, ist jedoch ein kostengünstiger, autarker Sensor nötig, der leicht zu montieren und zu betreiben ist. Im Projekt VITAL wurden neue Methoden zur LSA-Steuerung erprobt und entwickelt. Ein Ziel war die Untersuchung eines emissionsabhängigen Steuerungsverfahrens. Dabei wurde ein Sensor entwickelt, der Stickstoffoxide messen kann und in Echtzeit in einer Datenbank zur Verfügung stellt. Durch seine handelsüblichen Bauteile ist er leicht an bestehende Systeme anzupassen. Die Untersuchung der Schadstoffe in Abhängigkeit der Verkehrsstärke erfolgte auf der Messund Versuchstrecke des DLR in Berlin. Als Referenz dienten Doppelinduk- tionsschleifen. Im Laufe des Versuchs kam heraus, dass die gemessenen Schadstoffwerte mit der Verkehrsstärke korrelieren, jedoch nicht reproduzierbar sind und zu träge auf eine Änderung der Verkehrsstärke reagieren. Daher ist eine emissionsabhängige Verkehrssteuerung nur bedingt möglich. Der Sensor bietet allerdings die Möglichkeit die StickoxidBelastungen flächendeckend und aufwandsarm aufzuzeichnen.

\section{Einführung}

\subsection{Motivation}

Um das Jahr 1900 lebten etwa 165 Millionen Menschen in Städten. Das waren damals 10\% der Weltbevölkerung. Für das Jahr 2025 wird prognostiziert, dass etwa 4,5 Milliarden Menschen in Städten leben werden. Das sind bereits $57 \%$ der erwarteten Weltbevölkerung [Nies12] [Bund10]. Mit steigender Bevölkerungszahl steigt auch der Grad der Luftverschmutzung. Besonders in Ballungsräumen stellt dies ein immer größer werdendes Problem dar. Eine kostengünstige Lösung, um die Luftgüte engmaschig aufzeichnen zu können, könnte dabei helfen die Belastung zu reduzieren. 


\subsection{Anforderungen an den Sensor}

Im Projekt VITAL [Wire17][Oert16] bestand unter anderem die Frage, ob es möglich ist Ampelsteuerungen auf Grundlage der aktuellen lokalen Emissionsbelastung $\mathrm{zu}$ beeinflussen. Dafür wurde ein Sensor benötigt, der die Abgasbelastung in Abhängigkeit der Verkehrsstärke messen kann und die folgenden Anforderungen erfüllt (Tabelle 1).

Die kostengünstige Herstellung des Sensors wird durch den Einsatz handelsüblicher Bauteile erreicht. Gleichzeitig muss jedoch auf den Energieverbrauch geachtet werden. In Kombination mit einer Solarzelle und einem entsprechend dimensionierten Akku kann der Sensor energieautark sein. Die Anbindung an das Internet geschieht über ein 3G/2G-Modem. Geeignete Sensoren zur Messung verkehrsstärkeabhängiger Emissionen sind zu ermitteln.

\subsection{Stand der Technik}

Für die Überwachung der Luftgüte in Berlin wurden in der Stadt Messeinrichtungen verteilt [Sena17]. Diese messen Größen wie Kohlenstoffmonoxid, Stickoxide, Feinstaub und weitere. Diese Einrichtungen sind sehr groß und teuer im Unterhalt. Aus diesen Gründen sind sie ungeeignet. Vorteil ist jedoch die hohe Genauigkeit der Messwerte.

Die Firma Libelium aus Spanien stellt bereits Geräte her, die über ansteckbare
Sensoren unterschiedliche Umweltgrößen messen können. Die Waspmote Plug \& Sense! [Libe17] Serie wird über eine eigene IDE programmiert. Die Basis ist jedoch das Arduino Framework. Die Geräte sind kompliziert in der Programmierung und Tests haben gezeigt, dass die Genauigkeit der Messwerte nicht ausreicht. Darüber hinaus reicht die Energieversorgung über die mitgelieferte Solarzelle für den mitteleuropäischen Raum nicht aus.

In Stuttgart wird im Projekt 2MOVE2 [Land13] bereits eine emissionsabhängige Verkehrssteuerung erprobt. Dazu sollen zum einen die stationären Messungen der Landesanstalt für Umwelt, Messungen und Naturschutz Baden-Württemberg (LUBW) und zum anderen numerische Luftschadstoffsimulationen genutzt werden. Ein eigenständiger Sensor wird hier ebenfalls nicht genutzt.

\section{Entwicklung des Sensors}

Die Entwicklung des Sensors erfolgt in 2 Schritten. Im ersten Schritt werden die Funktionen hergestellt. Dazu gehören die Verbindung mit der Datenbank über eine Funk-

Verbindung, die Steuerung der Messelektronik und die Messwertaufnahme. Mit dem Sensor konnten nun verschiedene Gassensoren auf ihre Eignung getestet werden. Im zweiten Schritt wird der Sensor unter Berücksichtigung der Anforderungen aufgebaut.

\begin{tabular}{|l|l|}
\hline Anforderung & Priorität \\
\hline Kostengünstig in der Herstellung & Mittel \\
\hline Wetterfest (Spritzwasser- und Staubgeschützt) & Mittel \\
\hline Verkehrssärkeabhängige Emissionen messen & Hoch \\
\hline Stromsparend & Hoch \\
\hline Autark in den Punkten Energie und Kommunikation & Hoch \\
\hline Selbstorganisierend & Mittel \\
\hline Kleine Bauform & Niedrig \\
\hline
\end{tabular}




\subsection{Auswahl der Bauteile}

Als Plattform für den ersten Schritt wurde der Raspberry PI 2 gewählt. Auf dem Kleinstrechner arbeitet ein Debian Linux und er verfügt über eine gut ausgebaute Community. Für den zweiten Schritt ist ein Intel Edison geplant.

Für die Steuerung und Messelektronik wurde eine Leiterplatte entwickelt, die es ermöglicht verschiedene Sensoren anzuschließen und Messkreise anzupassen. Darüber hinaus befinden sich die Spannungsversorgungen für die einzelnen Baugruppen und ein I2C Bus und eine UART-Schnittstelle zum Modem.

\subsection{Auswahl der Messgrößen}

Für die Auswahl der Messgrößen kommen mehrere Stoffe in Frage. Bei der Verbrennung von Benzin und Diesel entstehen unterschiedliche Schadstoffe. In Abbildung 1 sieht man die Zusammensetzung der Abgase, die bei der Verbrennung von Benzin und Diesel entstehen. In beiden Fällen ist der Stickstoffanteil am größten mit rund 70\%. Stickstoff (N2) kommt jedoch schon zu $78 \%$ in der Luft vor, daher ist es ungeeignet als Messgröße. Der zweitgrößte Anteil in beiden Fällen ist Kohlenstoffdioxid mit $14 \%$ bzw. $12 \%$. Der drittgrößte Anteil ist Wasser (H2O) mit 13\% bzw.
11\%. Bei der Verbrennung von Diesel entsteht zusätzlich noch Sauerstoff (O2). Wie Stickstoff ist auch Sauerstoff (O2) zu 20\% Bestandteil der Luft. Es kommt daher auch nicht als Messgröße infrage. Die geringsten Anteile haben Kohlenstoffmonoxid (CO), Stickoxide (NOx) und Weitere.

Für die Auswahl der Messgrößen kommen daher Sensoren für Kohlenstoffmonoxid (CO), Kohlenstoffdioxid (CO2), Stickstoffmonoxid (NO) und Stickstoffdioxid (NO2) infrage.

Neben den Schadstoffen sind die Umweltgrößen Temperatur ( ), Luftfeuchte $(\varphi)$ und Luftdruck (p) auch Größen von Interesse. Zur Überwachung des Sensors werden die Batteriespannung und die Innentemperatur des Sensorgehäuses ebenfalls aufgezeichnet.

\section{Ergebnisse und Auswertung}

Für die Messungen wurde der Sensor in einem regengeschützten Gehäuse auf der Mess- und Versuchsstrecke des DLR auf dem Ernst-Ruska-Ufer in Berlin Adlershof aufgebaut. Als Referenzgröße wurde die Verkehrsstärke in Fahrzeuge pro Stunde gewählt. Dafür wurden die in der Straße verbauten Doppelinduktionsschleifen genutzt. Zu Spitzenzeiten

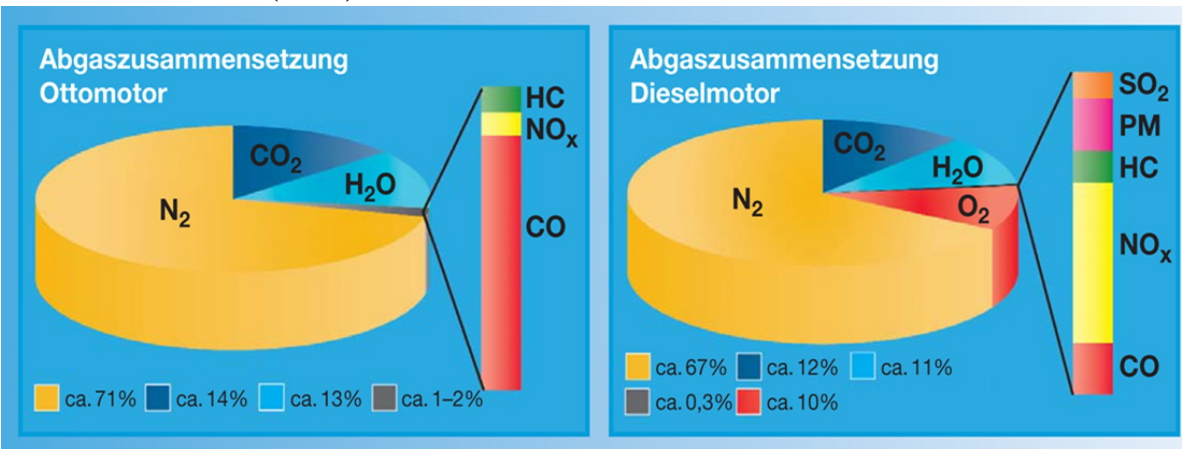

Abbildung 1: Abgaszusammensetzung von Otto- und Dieselmotoren aus [Wirt14] 
passieren etwa 1600 Fahrzeuge pro Stunde den Messquerschnitt. Die Messwerte werden in Spannung angegeben, da die Sensoren nicht kalibriert sind. Für den Versuch geht es darum, herauszufinden, ob die Messgrößen mit der Verkehrsstärke korrelieren und reproduzierbar sind. Die Messwerte werden im Minutentakt erhoben und bereits im Umweltsensor durch einen gleitenden Mittelwert geglättet. Die Fenstergröße ist 10. Der Wert hat sich als praktikabel erwiesen Spitzen aus den Messwerten zu entfernen. An die Datenbank werden die geglätteten und die Rohdaten übermittelt. verwendet. Der Korrelationskoeffizient am 01.10.16 ist $r=0,08$ und am 02.10.16 $r=0,045$. In diesem Fall kann man von keiner linearen Abhängigkeit sprechen. Im zweiten Versuch wurde der Sensor für Stickstoffmonoxid verwendet. Die Messwerte wurden über 4 Tage aufgenommen. Begonnen am Freitag den 21.10. bis Montag den 24.10. In Abbildung 3 ist der Verlauf für die NO-Belastung und die Verkehrsstärke für 2 aufeinander folgende Tage abgebildet (Samstag und Sonntag). Man sieht, dass die Messspannung bei steigender Verkehrsstärke steigt und bei sinkender Verkehrsstärke wieder fällt. Die gemes-

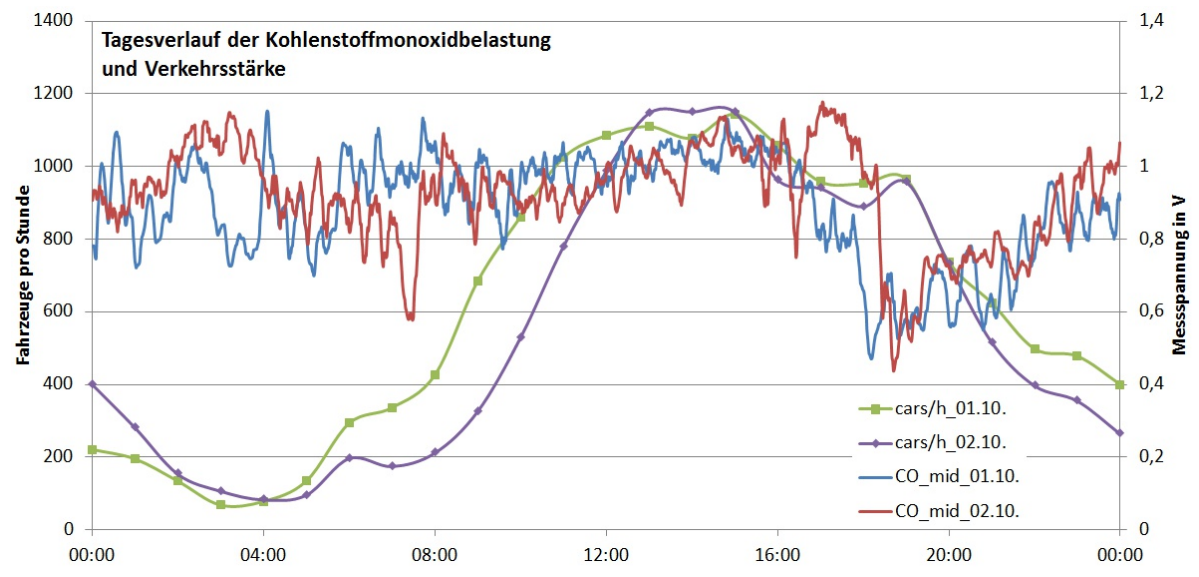

Abbildung 2: Tagesverlauf der Kohlenstoffmonoxidbelastung u. Verkehrsstärke 01. u. 02.10.16 (Sa u. So)

Der erste Versuch wurde mit dem COSensor durchgeführt. Abbildung 2 zeigt den Verlauf der Kohlenstoffmonoxidbelastung und Verkehrsstärke für 2 aufeinander folgende Tage. Man kann deutlich erkennen, dass der Verlauf der CO-Belastung an beiden Tagen einen ähnlichen Verlauf hat, jedoch nicht mit der Verkehrsstärke korreliert. Um die Eignung der Sensoren zu bewerten, wird der empirische Korrelationskoeffizient senen Werte korrelieren mit der Verkehrsstärke. Der

Korrelationskoeffizient beträgt für den 22.10. $r=0,474$ und für den 23.10. $r=0,627$. Die Differenz zwischen maximalem und minimalem Wert beträgt $\Delta \mathrm{U}=1,4 \mathrm{~V}$.

In Abbildung 4 ist der Tagesverlauf der Stickstoffmonoxidbelastung und der Verkehrsstärke für den 21. und 24.10.16 (Freitag und Montag) abgebildet. Am 


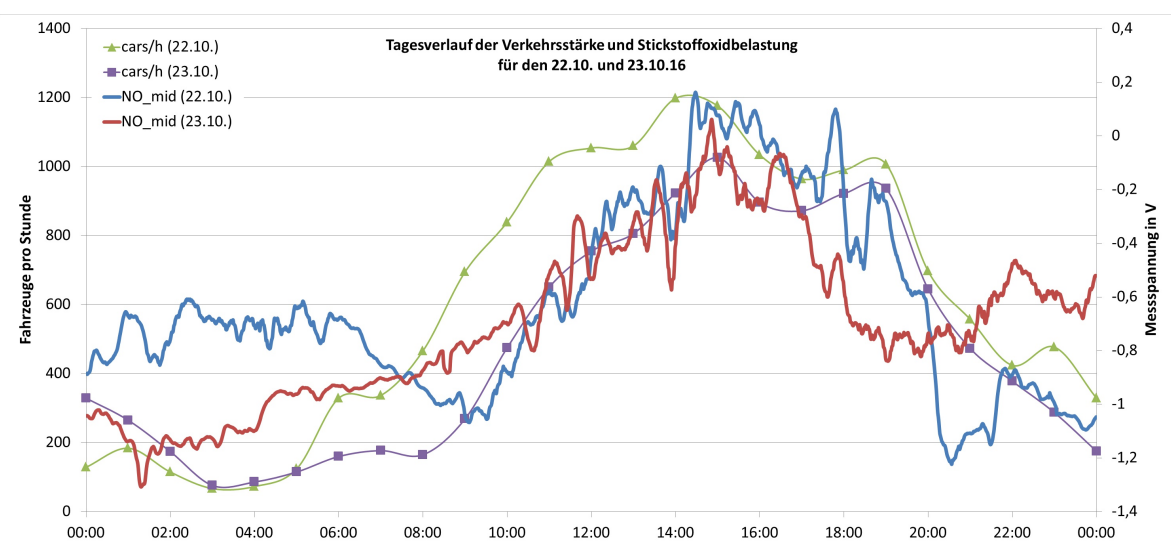

Abbildung 3: Tagesverlauf der Stickstoffmonoxidbelastung und Verkehrsstärke am 22. u. 23.10.16 (Sa u. So) 21.10. (blaue Kurve) ist eine Ab- Verlauf der NO2-Konzentration und hängigkeit zur Verkehrsstärke erkennbar. Verkehrsstärke für 2 aufeinander folDer Korrelationskoeffizient liegt bei gende Tage. Bei steigender $\mathrm{r}=0,588$. Der Korrelationskoeffizient für Verkehrsstärke steigt die Messspannung den 24.10. (rote Kurve) liegt bei $\mathrm{r}=$ - und bei fallender Verkehrsstärke sinkt 0,478. Eine Abhängigkeit zur Verkehrsstärke ist nicht erkennbar. Darüber hinaus liegen, trotz ähnlicher Verkehrsstärke, die Messspannungen für beide Tage im Mittel etwa 1,5V auseinander. Im dritten Versuch wurde der NO2Sensor verwendet. Abbildung 5 zeigt den die Messspannung wieder. Auch in diesem Fall hängt die Spannung von der Verkehrsstärke ab. Der Korrelationskoeffizient für den 22.10. beträgt $r=0,355$ und für den 23.10. $r=0,373$. Die Differenz zwischen Maximum und Minimum beträgt $\Delta \mathrm{U}=0,7 \mathrm{~V}$.

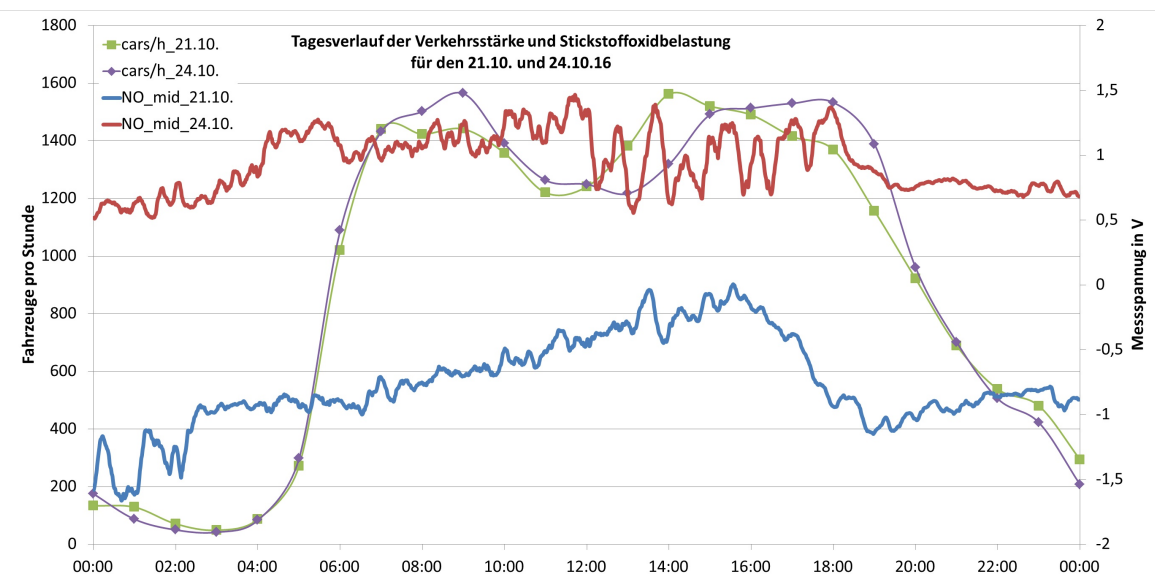

Abbildung 4: Tagesverlauf der Stickstoffmonoxidbelastung u. Verkehrsstärke am 21. u. 24.10.16 (Fr und Mo) 


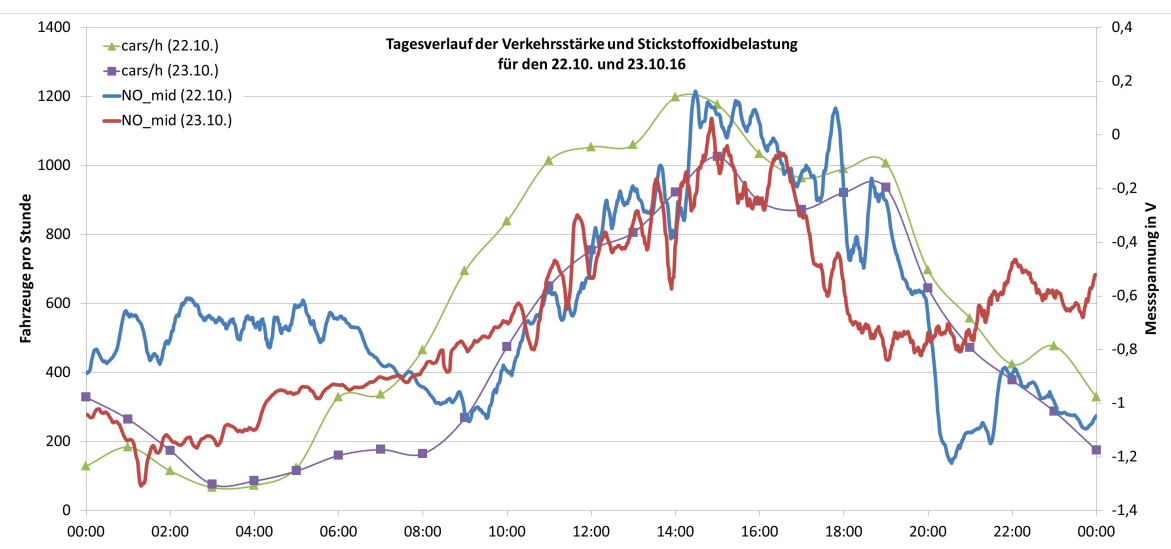

Abbildung 5: Tagesverlauf der Stickstoffdioxidbelastung und Verkehrsstärke am 22. u. 23.10.16. (Sa u. So)

In Abbildung 6 sind die Verläufe der Zusammenfassend kann man in Tabelle Stickstoffdioxidbelastung für den 21.10. 2 die Korrelationskoeffizienten der Gasund 24.10.16 (Freitag und Montag) und sensoren für den Messzeitraum sehen. In für die Verkehrsstärke abgebildet. Für den Versuchsvorbereitungen war bereits den 21.10. ist eine Abhängigkeit von der erkennbar, dass der CO-

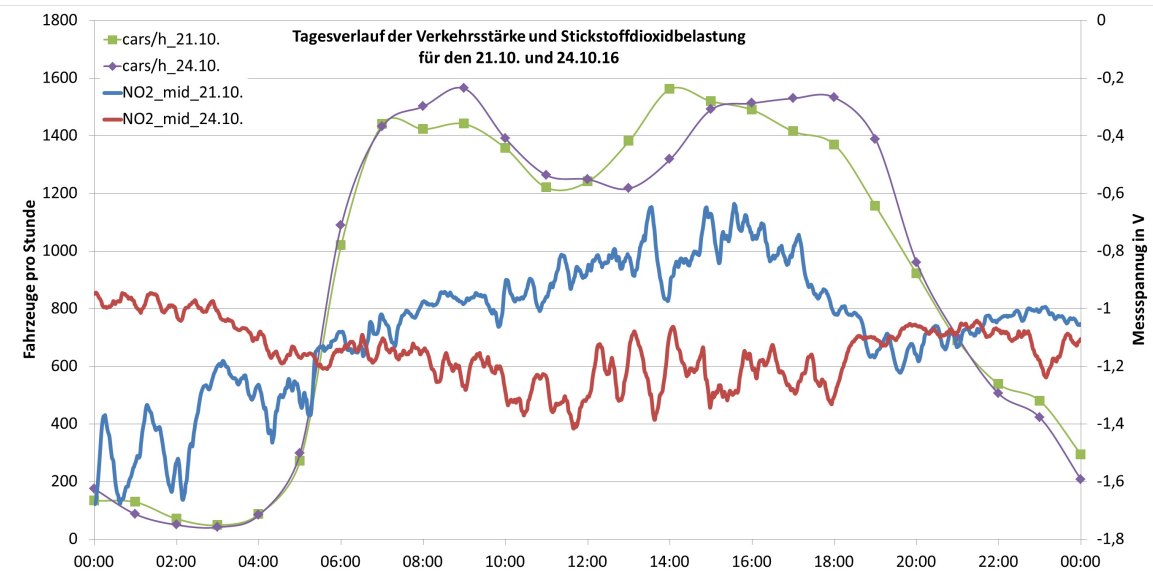

Abbildung 6: Tagesverlauf der Stickstoffdioxidbelastung und Verkehrsstärke am 21. und 24.10.16. (Fr u. Mo)

Verkehrsstärke erkennbar. Der Korrelationskoeffizient beträgt $r=0,639$. Wie bei der NO-Belastung am 24.10. ist auch bei der NO2-Belastung keine Abhängigkeit zur Verkehrsstärke erkennbar. Der Korrelationskoeffizient ist $r=-0,539$.
Sensor nicht geeignet ist. Daher wurde mit ihm nur an 2 aufeinander folgenden Tagen gemessen. Der Korrelations-koeffizient ist an beiden Tagen kleiner 0,1. Laut [Fern16] besteht eine schwache bis keine lineare Abhängigkeit zwischen 


\begin{tabular}{|l|l|l|l|}
\cline { 2 - 4 } \multicolumn{1}{c|}{} & \multicolumn{3}{l|}{ Korrelationskoeffizienten } \\
\hline Messung & $\mathrm{CO}$ & $\mathrm{NO}$ & $\mathrm{NO}_{2}$ \\
\hline Tag 1 & 0,08 & 0,588 & 0,639 \\
\hline Tag 2 & 0,045 & 0,474 & 0,355 \\
\hline Tag 3 & & 0,627 & 0,373 \\
\hline Tag 4 & & $-0,478$ & $-0,539$ \\
\hline mean & & 0,303 & 0,207 \\
\hline $\begin{array}{l}\text { mean } \\
\text { ohne Tag 4 }\end{array}$ & & 0,563 & 0,456 \\
\hline
\end{tabular}

Tabelle 2: Korrelationskoeffizienten der Gassensoren

den Messwerten und der Verkehrsstärke. Er kommt daher nicht infrage.

Der mittlere Korrelationskoeffizient für den NO-Sensor beträgt 0,303 bzw. 0,563.
In diesem Fall liegt eine schwache bis mittlere lineare Abhängigkeit vor. Der mittlere Korrelationskoeffizient für den NO2-Sensor beträgt 0,207 bzw. 0,456. Somit liegt eine schwache lineare Abhängigkeit vor.

Betrachtet man die gemessenen NO und $\mathrm{NO} 2$ Werte über den gesamten Messzeitraum (Abbildung 7), sieht man eindeutig einen sich wiederholenden Tagesverlauf. Eine Ausnahme bildet der 4. Tag. An ihm hängen die Messwerte für NO und NO2 nicht von der Verkehrs-

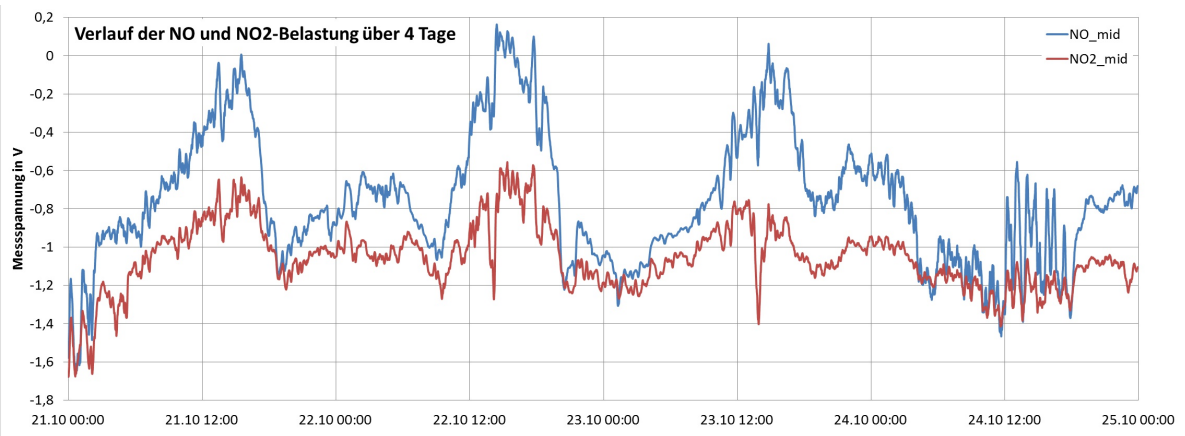

Abbildung 7: Verlauf der NO- und NO2-Belastung über den gesamten Messzeitraum

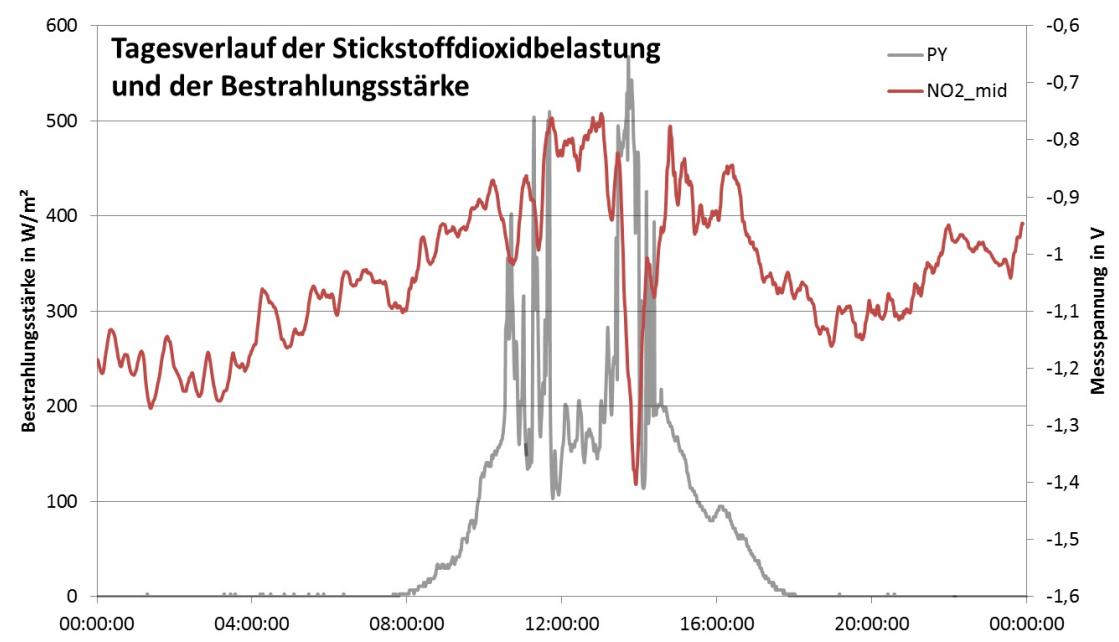

Abbildung 8: Tagesverlauf der Stickstoffdioxidbelastung und Bestrahlungsstärke durch die Sonne am 23.10 .16 
stärke ab. Darüber hinaus ergibt sich auch ein negativer Korrelationskoeffizient. Zu den lokalen Wetterdaten und den vom Sensor gemessenen Umweltdaten ergibt sich kein Zusammenhang. Der Messwerteverlauf am 24.10. bildet daher eine Ausnahme.

In Abbildung 5 ist bei ca. 14 Uhr eine Spitze in der Messspannung des NO2Sensors zu erkennen. Abbildung 8 zeigt denselben Messverlauf des NO2-Sensors zusammen mit der Bestrahlungsstärke am selben Tag. Deutlich zu erkennen ist, dass die Sonneneinstrahlung zu dieser Zeit bei ungefähr $500 \mathrm{~W} / \mathrm{m}^{2}$ lag. In dieser Zeit hat die Sonne den NO2-Sensor so erwärmt, dass der Sensorwiderstand angestiegen ist. Denselben Peak gibt es beim NO-Sensor auch, nur ist er nicht so stark ausgebildet.

\section{Fazit}

Im Verlauf der Untersuchungen kam heraus, dass Stickstoffmonoxid und Stickstoffdioxid geeignet sind verkehrsstärkeabhängige Emissionsbelastungen $\mathrm{zu}$ messen. Der NO-Sensor ist besser geeignet als der NO2-Sensor, da er eine stärkere lineare Abhängigkeit zur Verkehrsstärke aufweist und eine größere Differenz zwischen minimalem und maximalem Messwert hat. Jedoch gibt es Einflüsse, wie bspw. direkte Sonneneinstrahlung, die die Messwerte beeinflussen. Zusätzlich müssten die gemessenen Werte reproduzierbar sein, um für die emissionsabhängige Verkehrssteuerung geeignet zu sein. Dies hat sich jedoch nicht herausgestellt. Ein Kohlenstoffdioxidsensor wurde aus zeitlichen Gründen nicht untersucht. Seine Eignung sollte vor Beginn der 2. Entwicklungsphase untersucht werden.

Der Raspberry Pi 2 sowie die Funkverbindung zur Datenbank haben sich als zuverlässig erwiesen. In den Tests wurde 22 der Sensor mit 2 wieder aufladbaren Batterien mit je 144Wh betrieben. Dadurch war es möglich, auch ohne Solarzelle, über 6 Tage zu messen. Bei insgesamt 288Wh zur Verfügung stehender Energie und 144h Betriebszeit ergibt sich eine mittlere Leistungsaufnahme von $2 \mathrm{~W}$.

In den folgenden Schritten ist die Migration des Systems vom Raspberry PI 2 auf den Intel Edison vorzunehmen. Die Steuer- und Messplatine ist auf die wesentlichen Bestandteile $\mathrm{zu}$ reduzieren. Darüber hinaus ist die Dimensionierung und Integration der Solaranlage vorzunehmen.

\section{Literaturverzeichnis}

[Nies12]

Niesing Birgit: Die Zukunft der Stadt. In: weiter.vorn 4 (2012), S. 8-13.

[Bund10]

Statistisches Bundesamt: Globale Stadt- und Landbevölkerung in den Jahren 1995, 2010 und 2025 (in Millionen). 2010, https://de.statista.com/statistik/daten/studie/155946/umfrage/globale-bevoelkerung-stadt-und-land/. Abruf am 2017-01-05

[Oert16]

Oertel Robert1, Schnieder Lars1, Frankiewicz Tobias1, Trumpold Jan1, Krenkel Ralf2, Ruppe Sten1: Empirische Absicherung innovativer Steuerverfahren für Lichtsignalanlagen im öffentlichen Straßenraum. In: AAET2016, Deutsches Zentrum für Luft- und Raumfahrt e.V.1, BELLIS GmbH2, Braunschweig, 2016.

[Wire17]

Wired.de - Benedikt Plass-Fleßenkämper: Wie intelligente Ampeln Staus verhindern sollen, 2017, https://www.wired.de/collection/tech/wie-intelligente-ampeln-staus-verhindern-sollen?utm_campaign $=$ Daily + Bits $+-+15.02 .2017 \& u$ tm_source $=\mathrm{N}$ ewsletter\&utm_medium=email\&utm_campaign $=$ Wired $\% 3 \mathrm{~A}+$ Daily + Bits\&utm_medium $=$ email\&utm_source=wired. Abruf am 2017-02-15 
[Sena17]

Senatsverwaltung für Umwelt, Verkehr und Kli-

maschutz Berlin: BLUME-Messnetz und

Luftdaten, 2017,

http://www.stadtentwicklung.berlin.de/um-

welt/luftqualitaet/luftdaten/index.shtml. Abruf

am 2017-02-13

[Libe17]

Libelium Communicaciones Distribuidas S.L.:

Waspmote Plug \& Sense! Technical Guide, 2017, http://www.libelium.com/development/plugsense /documentation/waspmote-plug-sense-technicalguide/?action=download. Abruf am 2017-02-10

[Land13]

Landeshauptstadt Stuttgart: 2MOVE2 - Nachhaltiger Stadtverkehr in europäischen Städten, 2013, https://www.stuttgart.de/2move2. Abruf am 2017-02-13

[Wirt14]

Wirtschaftsgesellschaft des Kraftfahrzeuggewerbes mbH: Faszination Chemie - interaktive Beispiele aus der Lebenswirklichkeit für die Schule, 2014, http://www.chemie-am-auto.de/abgase/. Abruf am 2017-01-19

\section{[Fern16]}

Fernuniversität in Hagen: Streudiagramme und Korrelation - Korrelationskoeffizient, 2016, http://www.fernuni-hagen.de/ksw/neuestatistik/content/MOD_23196/html/comp_23234.html. Abruf am 2017-01-31

\footnotetext{
(CC)
} 